\title{
Fortsetzung von Donepezil verhindert Pflegeheimeinweisung
}

Fragestellung: Die Studie untersucht, ob die Fortsetzung von Donepezil und/oder zusätzliche Gabe von Memantin bei Patienten mit mittelschwerer bis schwerer Demenz vom AlzheimerTyp, die über einen Zeitraum von mindestens drei Monaten kontinuierlich mit Donepezil behandelt waren, die Einweisung in ein Pflegeheim beeinflusst.

Hintergrund: Die Donepezil and Memantine in Moderate to Severe Alzheimer's Disease (DOMINO-AD)-Studie hat in der Vergangenheit gezeigt, dass die Fortführung einer Donepezil-Therapie bei Patienten mit mittelschwerer bis schwerer Demenz vom Alzheimer-Typ die kognitive Leistungsfähigkeit, gemessen am Mini-Mental-State-Examination-Test, und Aktivitäten des täglichen Lebens, gemessen an der Bristol Activities of Daily Living Scale, im Vergleich zu Patienten, bei denen Donepezil abgesetzt wurde, günstig beeinflusst. Das Ausmaß der Verbesserung der Kognition und der funktionellen Verbesserung im Alltagsleben war mit 1,9 Punkten (95\%-Konfidenzintervall [KI] 1,3-2,5) und 3,0 Punkten (95\%-KI 1,8-4,3) moderat, weshalb der tatsächliche Nutzen der Medikamentenfortsetzung in Frage gestellt worden war. Im Rahmen einer sekundären und Posthoc-Analyse wurde nun untersucht, in welcher Weise die Fortsetzung des Donepezils beziehungsweise die zusätzliche Gabe des Memantins die Einwei-

Howard R, McShane R Lindesay J et al. Nursing home placement in the Donepezil and Memantine in Moderate to Severe Alheimer's Disease (DOMINO-AD) trial: secondary and post-hoc analyses. Lancet Neurol 2015; 14: $1171-81$ sung in Pflegeeinrichtungen beeinflusst.

Patienten und Methodik: In die randomisierte doppelblinde placebokontrollierte Studie wurden 295 Patienten mit Mini-Mental-State-Examination-Score von $15-13$
Punkten unter mindestens dreimonatiger Behandlung mit $10 \mathrm{mg}$ Donepezil hinsichtlich der unveränderten Fortführung der Donepezil-Therapie, der Fortführung der Donepezil-Therapie mit zusätzlicher Verordnung von $20 \mathrm{mg}$ Memantin täglich, des Absetzens des Donepezils und des Absetzens des Donepezils mit Verordnung von $20 \mathrm{mg}$ Memantin täglich randomisiert. Die Behandlung gemäß Studienprotokoll wurde über einen Zeitraum von 52 Wochen durchgeführt, anschließend wurde die Behandlungsentscheidung den zuständigen Ärzten überlassen. Pflegeheimeinweisungen wurden über die 52-wöchige Studienphase und anschließend in 26-wöchigen Abständen über weitere drei Jahre erfasst.

Ergebnisse: In einer im Studienprotokoll prädefinierten Analyse fiel auf, dass sich die Zeitlatenz zur Einweisung in ein Pflegeheim zwischen Patienten, bei denen Donepezil abgesetzt wurde, und solchen, bei denen Donepezil fortgeführt wurde, nicht signifikant unterschied $(p=0,100)$. Die angesichts des statistischen Trends durchgeführten nachfolgenden, im Studienprotokoll nicht prädefinierten Post-hoc-Analysen zeigten, dass das Risiko einer Einweisung in eine Pflegeeinrichtung im ersten Studienjahr durch Absetzen des Donepezils signifikant erhöht war (Hazard-Ratio [HR] 2,09; 95\%-KI 1,29-3,39), wohingegen sich das Pflegeheimeinweisungsrisiko nach vier Jahren erneut nicht unterschied (HR 0,89; $95 \%$-KI 0,58-1,35]). Die Neuverordnung des Memantins hatte keinerlei Einfluss auf das Risiko einer Pflegeheimeinweisung (HR 0,92; $95 \%$-KI 0,58-1,45) nach einem Jahr und nach vier Jahren (HR 1,23; $95 \%$-KI 0,81-1,87).

Schlussfolgerungen: Die Autoren folgern, dass die Fortsetzung der Donepezil-Therapie angesichts der Verzögerung der Pflegeheimeinweisung kosteneffektiv und für die Lebensqualität der Patienten bedeutsam ist.

\section{- Kommentar von Dirk Hermann, Essen}

\section{Die Fortsetzung der Therapie ist gerechtfertigt}

Beobachtungen geringer Effektstärken pharmakologischer Behandlung mit Cholinesteraseinhibitoren und Memantin haben in der Vergangenheit die Frage nach minimal zu fordernden Effektgrößen, welche für das Alltagsleben der betroffenen Patienten bedeutsam sind, aufgeworfen. In der vorliegenden Studie wurde ein harter Endpunkt analysiert, der zweifelsfrei für die Lebensqualität der Patienten relevant ist.

Einschränkend muss festgehalten werden, dass es sich bei der Datenanalyse um Post-hoc-Analysen handelt. Die Daten rechtfertigen die Fortsetzung des Donepezils bei Patienten mit fortgeschrittener Demenz.

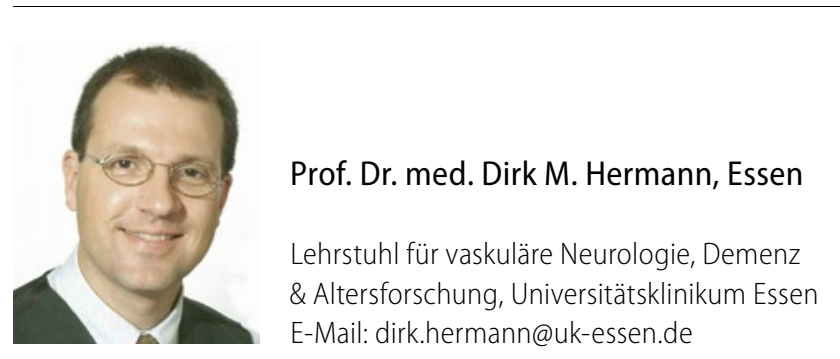

